\title{
Objekttyp: FrontMatter
}

\section{Zeitschrift: Schweizerische Zeitschrift für Geschichte = Revue suisse d'histoire = Rivista storica svizzera}

Band (Jahr): 69 (2019)

Heft 1

PDF erstellt am:

26.04.2023

\section{Nutzungsbedingungen}

Die ETH-Bibliothek ist Anbieterin der digitalisierten Zeitschriften. Sie besitzt keine Urheberrechte an den Inhalten der Zeitschriften. Die Rechte liegen in der Regel bei den Herausgebern.

Die auf der Plattform e-periodica veröffentlichten Dokumente stehen für nicht-kommerzielle Zwecke in Lehre und Forschung sowie für die private Nutzung frei zur Verfügung. Einzelne Dateien oder Ausdrucke aus diesem Angebot können zusammen mit diesen Nutzungsbedingungen und den korrekten Herkunftsbezeichnungen weitergegeben werden.

Das Veröffentlichen von Bildern in Print- und Online-Publikationen ist nur mit vorheriger Genehmigung der Rechteinhaber erlaubt. Die systematische Speicherung von Teilen des elektronischen Angebots auf anderen Servern bedarf ebenfalls des schriftlichen Einverständnisses der Rechteinhaber.

\section{Haftungsausschluss}

Alle Angaben erfolgen ohne Gewähr für Vollständigkeit oder Richtigkeit. Es wird keine Haftung übernommen für Schäden durch die Verwendung von Informationen aus diesem Online-Angebot oder durch das Fehlen von Informationen. Dies gilt auch für Inhalte Dritter, die über dieses Angebot zugänglich sind. 


\section{SCHWEIZERISCHE ZEITSCHRIFT FÜR GESCHICHTE} REVUE SUISSE
D'HISTOIRE

\section{RIVISTA STORICA SVIZZERA}

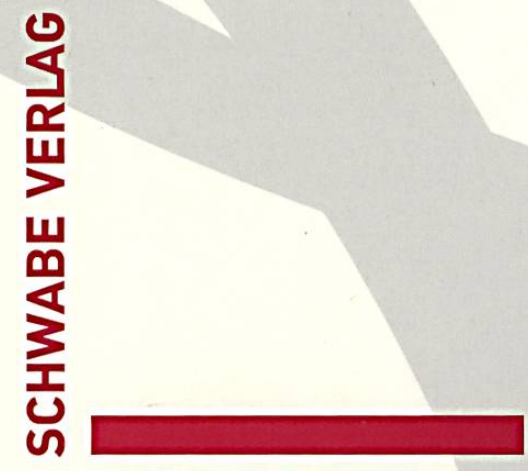


Die Schweizerische Zeitschrift für Geschichte (SZG) wird von der Schweizerischen Gesellschaft für Geschichte (SGG) herausgegeben und erscheint dreimal jährlich. Das Abonnement ist im Mitgliederbeitrag der SGG inbegriffen.

Die Qualität der wissenschaftlichen Artikel wird durch ein externes Peer ReviewVerfahren gesichert. Für die Aufnahme von Beiträgen ist die Redaktion zuständig. Für die Eingabe von Manuskripten siehe www.szg-rsh.ch.

In der SZG werden hauptsächlich Werke zur Geschichte der Schweiz sowie von in der Schweiz tätigen Historikerinnen und Historikern rezensiert. Der Rezensionsteil wird durch das SGG-Generalsekretariat koordiniert: rezensionen@szg-rsh.ch.

La Revue suisse d'histoire (RSH) est éditée par la Société suisse d'histoire (SSH) et parait trois fois par année. L'abonnement est compris dans la cotisation à la SSH.

La qualité des articles scientifiques est assurée grâce à un processus de peer review externe. La rédaction effectue la sélection des contributions. Pour le dépôt des manuscrits, cf. www.szg-rsh.ch.

Dans la RSH sont recensés principalement les travaux sur l'histoire suisse, ainsi que les travaux des historiennes et historiens actifs en Suisse. Les comptes rendus sont coordonnés par le Secrétariat général de la SSH: recensions@szg-rsh.ch.

La Rivista storica svizzera (RSS) è pubblicata dalla Società svizzera di storia (SSS) tre volte all'anno. Labbonamento è incluso nella quota d'associazione.

La qualità degli articoli scientifici è garantita da un processo di peer review esterno. La redazione decide sulla pubblicazione dei contributi. Per l'inoltro degli articoli cfr. www.szg-rsh.ch.

Nella RSS vengono recensite principalmente opere sulla storia della Svizzera e opere di storiche e storici attivi in Svizzera. Le recensioni vengono coordinate dalla Segreteria generale della SSS: recensioni@szg-rsh.ch.

ISSN $0036-7834$

Verlag/Maison d'édition/Casa editrice:

Schwabe Verlag, Schwabe Verlagsgruppe AG, Basel, www.schwabeverlag.ch

Unterstützt durch die Schweizerische Akademie der Geistes- und Sozialwissenschaften

www.sagw.ch 